\title{
UNIQUENESS OF A CLASS OF STEADY PLANE GRAVITY FLOWS
}

\author{
D. S. CARTER
}

1. Introduction. In a previous communication [2] we established the existence of a class of free boundary flows of an incompressible inviscid fluid in a uniform gravitational field, having plane projections as illustrated in Figure 1. The flow domain is bounded on the right

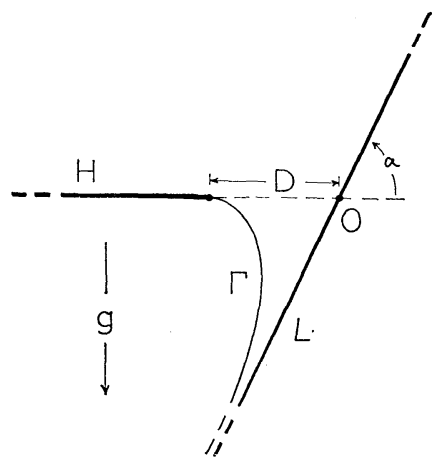

Fig. 1

by an infinite line $L$ inclined at an angle $\alpha$ with the horizontal, and below and on the left by a semi-infinite horizontal line $H$ and a free boundary arc $\Gamma$ joined to $H$. The flow is downward through the slot between $H$ and $L$ into a jet bounded by $L$ and $\Gamma$. The physical parameters include the boundary inclination $\alpha$, the acceleration of gravity $g$, the solt width $D$, the area flow rate (flux) $A$, and the flow speed at the slot edge, $q$. It is shown in [2] that there exists a similarity class of such flows for each choice of the dimensionless pair $\alpha, g A / q^{3}$ such that $0<\alpha<\pi, 0<g A / q^{3}<\infty$. The flows established there have convex free boundaries in the sense that their inclinations decrease monotonically from zero at the slot edge to $-\pi+\alpha$ at infinity, where they are asymptotic to the fixed boundaries $L$.

In this paper we shall establish the uniqueness of these similarity classes, find the ranges of the dimensionless products

$$
\kappa=q D / A, \quad \lambda=g A / q^{3}, \quad \mu=g D / q^{2}, \quad \nu=g D^{3} / A^{2}
$$

and determine the continuity and monotonicity relations between these products and other similarity invariants. (The products $\kappa, \lambda, \mu, \nu$ are singled out since each involves only three of the four quantities $g, D$,

Received October 30, 1963. Work partially supported by U. S. National Science Foundation Grant GP 141. 
$A, q$. Notice that $\lambda$ has been changed by the factor $\pi / 6$ from its value in [2]; this change does not affect our qualitative results.) The uniqueness and monotonicity theorems are independent of the existence theorems of reference [2]. They are based on the Lavrentieff-Serrin theorems summarized in $\S 2$. They also depend on the asymptotic jet behavior established in [4], although only on the "asymptotic free boundary shape" as expressed by equation (1.3) below.

We assume specifically that

(a) each flow occupies a simply-connected schlicht domain bounded by $L$ and $H+\Gamma$

(b) the complex velocity potential $W=U+i V$ maps this domain conformally onto the infinite strip $0<V<A$ so that $H+\Gamma$ goes into $V=0$ and $L$ goes into $V=A$

(c) the flow velocity exists on $\Gamma$, including the endpoint on $H$, as a limit from within the flow domain

(d) $\Gamma$ extends downward to infinity in the sense that the vertical depth $h$ below $H$ tends to $+\infty$ on this curve

(e) the condition of constant pressure, relating the flow speed $v$ and depth $h$, is satisfied on $\Gamma$ :

$$
v^{2}=q^{2}+2 g h
$$

Stronger explicit restrictions than $(c)$ and $(d)$ on the asymptotic jet behavior are unnecessary because, as shown in [4], the conditions (a)-(e) together fix this behavior very closely. In particular the asymptotic shape of $\Gamma$, in terms of rectangular coordinates with positive $x$-ax is downward along $L$, is given by

$$
x y^{2} \sim A^{2} / 2 g \cos \alpha .
$$

The class of flows satisfying these conditions is invariant under a group of plane Euclidean motions as well as a dimensional similarity group (homotheties of the length and time scales). We eliminate the Euclidean motions by fixing once and for all the direction of gravity and position of the point $O$ on the slot at $L$.

Let $\mathscr{F}$ denote the family of all flows so determined, satisfying conditions $(a)-(e)$. This family remains invariant under the dimensional similarity group, which decomposes $\mathscr{F}$ into minimal invariant subsets-the similarity classes. A general form of the uniqueness theorem, established in $\S 4$, is

THEOREM 1.1. There is at most one similarity class in $\mathscr{F}$ having prescribed values of $\alpha$ and any one of the four products $\kappa, \lambda, \mu, \nu$.

As a direct corollary we have 
THEOREM 1.2. There is at most one flow in $\mathscr{F}$ having prescribed values of $\alpha$ and any three of the four quantities $g, D, A, q$. All such flows belong to the class established in [2].

For each value of $\alpha$ the products $\kappa, \lambda, \mu, \nu$ are shown in $\S 4$ to be continuous monotonic functions of one another such that $\kappa$ decreases as $\lambda, \mu, \nu$ increase. The ranges of $\lambda, \mu, \nu$ are all $(0, \infty)$ while $\kappa$ is restricted to an interval $\left(\kappa_{\infty}, \kappa_{0}\right), 0<\kappa_{\infty}<\kappa_{0}<\infty$, whose limits depend on $\alpha$. These bounds on $\kappa$, and the limiting flows to which they correspond, are determined in $\S 5$.

The fact that for fixed $\alpha$ the ranges of $\mu$ and $\nu$ are both $(0, \infty)$ implies the existence, as well as the uniqueness, of a flow in $\mathscr{F}$ having arbitrarily given geometric parameters $\alpha, D$, as well as $g$ and either $A$ or $q$. Not all these flows can be expected to be stable. It is intuitively very plausible that they are stable for small, but unstable for high, values of $\lambda, \mu, \nu$. (Thus let $\alpha, D, g$ be fixed. When $A$ and $q$ are large the fluid is forced rapidly through the solt and small disturbances wash quickly downstream into the jet. When $A$ and $q$ are small the flow proceeds slowly across the slot, where $\Gamma$ is almost horizontal before turning abruptly as it nears $L$ (see $\S 5$ ) ; the limiting flow has a stationary horizontal free boundary which is certainly unstable.)

For the proofs we shall work entirely with families of dimensionless flows. Except as otherwise noted in $\$ \S 4,5$, all flows are assumed to belong to the family $\mathscr{F}_{1}$ obtained from $\mathscr{F}$ by taking $D$ and $D^{2} / A$ as units of length and time, respectively. The members of $\mathscr{F}_{1}$ are in 1:1 correspondence with the similarity classes in $\mathscr{F}$. They are defined in terms of the parameters $\alpha, \kappa, \nu$ alone, for the substitution $A=D=1$ in (1.1) yields $q=\kappa, g=\nu$, (in view of Theorem 1.1 only one of the parameters $\kappa$ and $\nu$ is actually needed to determine the flow, but until this is proved we may think of both $\kappa$ and $\nu$ together as defining the flow). With the above normalizations, the constant pressure condition (1.2) becomes

$$
v^{2}=\kappa^{2}+2 \nu h
$$

while the asymptotic free boundary shape (1.3) reduces to

$$
x y^{2} \sim(2 \nu \cos \alpha)^{-1} .
$$

The other products are determined by the identities

$$
\lambda=\nu / \kappa^{3}, \mu=\nu / \kappa^{2} .
$$

2. Comparison theorems. Here we collect several LavrentieffSerrin comparison theorems needed in the proofs. The terminology is as follows: A strip flow is one in which the complex velocity potential 
$W=U+i V$ is onto an infinite strip $0<V<A$; a half-plane flow is one in which $W$ is onto the half-plane $0<V<\infty$. A regular boundary point is a boundary point of the flow domain at which the flow velocity $u=\lim W^{\prime}$ exists as a limit from the interior and does not vanish.

THEOREM 2.1. Consider two flows with complex velocity potentials $W_{1}, W_{2}$, respectively, such that the domain of the second flow is contained in that of the first. Suppose that the streamlines $V_{1}=0$, $V_{2}=0$ have a common point $M$ at which the velocities of both flows exist as limits from within their domains. If either

(a) both are half-plane flows such that $W_{1}^{-1}(\infty)=W_{2}^{-1}(\infty)=\infty$, having $\infty$ as a regular boundary point and having equal velocities at $\infty$, or

(b) both are strip flows of equal flux $A$, and the streamline $V_{2}=A$ is part of the streamline $V_{1}=A$, then the respective flow speeds $v_{1 M}$, $v_{2 M}$ at $M$ satisfy

$$
v_{1 M} \geqq v_{2 M} \text {. }
$$

Equality holds if and only if the flows are identical or $v_{1 M}=0$.

THEOREM 2.2. Consider two strip or half-plane flows with complex velocity potentials $W_{1}, W_{2}$, respectively. Suppose that the flow domains contain a common simply-connected domain $\mathscr{D}$ having distinct boundary points $M, N$ which are regular boundary points of both flows. If either

(a) the boundary of $\mathscr{D}$ consists of a simple closed curve QMNQ, positively oriented around $\mathscr{D},(Q$ may be at infinity) such that $Q M N$ is part of the streamline $V_{1}=0$ and $M N Q$ is part of the streamline $V_{2}=0$, or

(b) both are strip flows of flux $A_{1}, A_{2}$, respectively, and the boundary of $\mathscr{D}$ consists of a simple closed curve $Q M N P Q$, positively oriented around $\mathscr{D}$, ( $P$ or $Q$ may be at infinity) such that $Q M N$ is part of the streamline $V_{1}=0, M N P$ is part of the streamline $V_{2}=0$, and $P Q$ is a common part of the streamlines $V_{1}=A_{1}, V_{2}=A_{2}$, then the respective flow speeds at $M$ and $N$ satisfy

$$
v_{1 N} / v_{1 M} \geq v_{2 N} / v_{2 M} .
$$

Equality holds if and only if the flows are geometrically similar (in the sense that $\left.W_{2}=a W_{1}+b\right)$.

Theorem 2.1 is essentially Theorem 1 of [7] combined with the first Comparison Theorem of [9] (Appendix), while Theorem 2.2 combines Serrin's "under-over theorems" $[7,8]$. Theorem $2.2(a)$ is stated so 
as to allow the simultaneous comparison of a strip flow with a halfplane flow as needed is $\S 3$; the proof is the same as that of Theorem 2 of [7].

3. Qualitative features. In preparation for the uniqueness proof we shall establish some qualitative flow features, including the fact that the free boundary cannot rise above the horizontal fixed boundary. Although proved for the "dimensionless family" $\mathscr{F}_{1}$, these results hold equally for $\mathscr{F}$.

LEMMA 3.1. There are no siagnation points on $\Gamma$.

Proof. To obtain a lower bound on the flow speed on $\Gamma$ note, in view of the constant pressure condition (1.2), that a point of minimum speed is also a point of minimum depth (i.e., maximum height above $H$ ). Let $N$ be any point of maximum height on $\Gamma$ and $P$ be the point on $L$ at the same height as $N$ (see Figure 2). Then the infinite sector

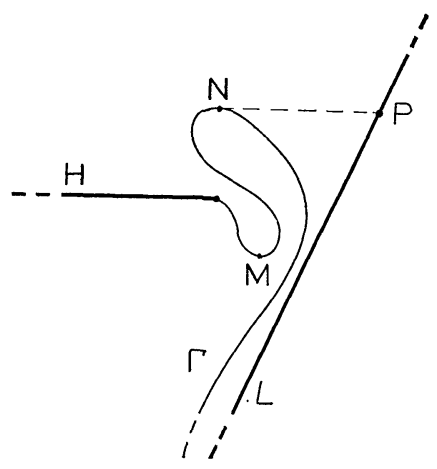

Fig. 2

to the left of $L$ and above the horizontal line through $N$ and $P$ lies inside the flow domain. Comparison with the "wedge flow" of unit flux in this sector, having a source at $\infty$ and a sink at $P$, using Theorem 2.1 (b), shows that the speed $v_{N}$ at $N$ satisfies

$$
v_{N}>(\pi-\alpha) / d
$$

where $d$ is the distance from $N$ to $P$.

Lemma 3.2. $\Gamma$ is an analytic arc except at the slot edge, where it is tangent to $H$.

Proof. Lewy has shown [5] that the free boundary is analytic except at endpoints and stagnation points. Moreover the boundary 
has a tangent wherever the flow velocity is defined and not zero. Thus the lemma follows by Lemma 3.1 and condition (c) on $\mathscr{F}$. (For further information on the flow behavior near the slot edge, see [3]).

Lemma 3.3. Except at the slot edge, $\Gamma$ lies entirely below $H$.

Proof. Let the points $N$ and $P$ be as in the proof of Lemma 3.1 and $M$ be a point of minimum height on that part of $L$ joining $H$ to $N$. We are to show that $N$ is at the slot edge, but it is sufficient to show that $M$ is at the same height as $N$. For then they are both level with $H$, and if $N$ is not at the slot edge, $\Gamma$ is initially an extension of the line $H$. Since $\Gamma$ is analytic (Lemma 3.2) it must itself be an extension of $H$, contradicting condition (d) on $\mathscr{F}$.

Suppose $N$ lies above $M$. We will show that the speeds $v_{M}, v_{N}$ at these points satisfy $v_{N}>v_{M}$, which contradicts the constant pressure condition (1.2). Consider two cases according as the inclination $\gamma$ of the segment joining $M$ and $N, 0<\gamma<\pi$, does or does not exceed $\alpha$. If $\gamma>\alpha$, let $L_{1}$ be a line parallel to $L$ lying sufficiently far to the left so as to intersect $H$ but not $\Gamma$ (see Figure 3). Consider a half-

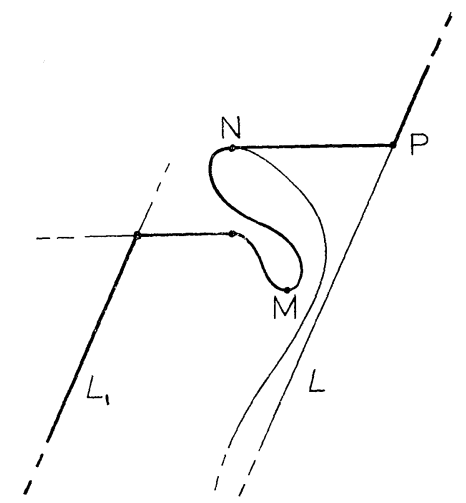

Fig. 3

plane flow in the domain $\mathscr{D}^{*}$ having as positively oriented boundary that part of $L_{1}$ below $H$, the segment of $H$ between $L_{1}$ and the slot edge, that part of $\Gamma$ between the slot edge and $N$, the horizontal segment $N P$, and the part of $L$ above $P$, whose complex velocity potential $W^{*}$ satisfies $W^{*}(\infty)=\infty$. Each of the three boundary points $M, N, \infty$, is a regular boundary point since the boundary is piecewise analytic with continuous inclination [6]. If $v_{\boldsymbol{M}}^{*}, v_{N}^{*}$ are the speeds of this flow at $M, N$, then it follows from Theorem 2.2 (a), with $Q=\infty$, that

$$
v_{N} / v_{M}>v_{N}^{*} / v_{M}^{*} .
$$


Now let this half-plane flow in $\mathscr{D}^{*}$ be translated along the segment $M N$ so that $M$ is brought into coincidence with $N$. Since $\gamma>\alpha$ the translated domain $\mathscr{D}^{* *}$ is inside $\mathscr{D}^{*}$, and Theorem 2.1 (a) yields $v_{N}^{*}>v_{\mu}^{*}$; hence $v_{N}>v_{\mu}$.

If $\gamma \leqq \alpha$, let $\mathscr{D}^{*}$ be the domain whose positively oriented boundary consists of $H$, the part of $\Gamma$ between the slot-edge and $N$, the segment $N P$, and the part of $L_{1}$ above $P$. Let $v_{\mu}^{*}, v_{N}^{*}$ be the flow speeds at $M, N$ of a strip flow in $\mathscr{D}^{*}$ with source at infinity and sink at $P$. The inequality (3.2) now follows on taking $Q=\infty$ in Theorem $2.2(\mathrm{~b})$. Finally let $\mathscr{D}^{* *}$ be the domain inside $\mathscr{D}^{*}$ obtained by translating $\mathscr{D}^{*}$ upward along $L$ until $M$ is raised to the same height as $N$, then contracting uniformly with respect to $P$ to bring the image of $M$ into coincidence with $N$. The flow in $\mathscr{D}^{* *}$ clearly has a complex potential of the form $W^{* *}(z)=W^{*}(a z+b),|a| \geqq 1$. It follows from Theorem 2.1 (b) that $v_{N}^{*}>|a| v_{M}^{*} \geqq v_{M}^{*}$; hence $v_{N}>v_{M}$ in this case also.

4. Uniqueness, monotonicity, and continuity. The proof of uniqueness hinges on

LEMma 4.1. Consider two flows belonging to the family $\mathscr{F}_{1}$ and having equal values of $\alpha$. Let the parameters $\kappa, \nu$ have the values $\kappa_{1}, \nu_{1}$ for one of these flows and $\kappa_{2}, \nu_{2}$ for the other. If $\nu_{1} \geqq \nu_{2}$ then $\kappa_{1} \leqq \kappa_{2}$, and equality holds only if the flows are identical.

Proof. Consider first a single flow in $\mathscr{F}_{1}$. In terms of polar coordinates $(r, \theta)$ with origin at $O$ and polar axis downward along $L$ the asymptotic free boundary shape (1.5) becomes

$$
\theta^{2} r^{3} \sim(2 \nu \cos \alpha)^{-1} .
$$

For each value of $\theta,-\alpha \leqq \theta<0$, the polar ray of inclination $\theta$ intersects $\Gamma$ at least once. Let $r_{\min }(\theta)$ and $r_{\max }(\theta)$ be the least and greatest distances, respectively, of such intersections from $O$. As functions of $\theta, r_{\min }$ and $r_{\max }$ are respectively lower and upper semi-continuous, and $r_{\min }$ has a positive lower bound. By Lemma 3.3,

$$
r_{\min }(-\alpha)=r_{\max }(-\alpha)=1 .
$$

Both functions satisfy (4.1) near $\theta=0$.

Now consider two flows in $\mathscr{F}_{1}$ with $\nu_{1} \geqq \nu_{2}$. Let $\rho=r_{\max 1} / r_{\min 2}$ be the ratio of $r_{\max }$ for the first flow to $r_{\min }$ for the second. Then $\rho$ is upper semi-continuous, is equal to 1 at $\theta=-\alpha$, and approaches $\left(\nu_{2} / \nu_{1}\right)^{1 / 3} \leqq 1$ as $\theta$ approaches 0 . Therefore $\rho$ attains its supremum $\rho_{0}$ at some point $\theta_{0},-\alpha \leqq \theta_{0}<0$. Let $N_{1}, N_{2}$ be the boundary points $\left(r_{\max 1}\left(\theta_{0}\right), \theta_{0}\right),\left(r_{\min 2}\left(\theta_{0}\right), \theta_{0}\right)$ of the respective flows, and $v_{1}, v_{2}$ be the 
corresponding flow speeds at these points. Since $N_{1}$ either coincides with $N_{2}$ or lies below it, and $\nu_{1} \geqq \nu_{2}$, the constant pressure condition (1.4) implies

$$
v_{1}^{2}-\kappa_{1}^{2} \geqq v_{2}^{2}-\kappa_{2}^{2}
$$

Now let the first flow be contracted uniformly about $O$ by the factor $\rho_{0}$ (its complex potential $W_{1}(z)$ replaced by $W_{1}\left(\rho_{0} z\right)$ ). The contracted domain is then inside that of the second flow, the boundaries being tangent at $N_{2}$; hence by Theorem 2.1 (b),

$$
v_{2} \geqq \rho_{0} v_{1} \geqq v_{1} \text {. }
$$

It follows that $\kappa_{1} \leqq \kappa_{2}$. Equality holds only if $\rho_{0}=1$ and the flows are identical.

(Notice that the above proof yields $\rho_{0}=1$ if the flows are identical. Hence each flow domain is star-shaped with respect to the origin-a fact which also follows once uniqueness has been proved, since the solutions established in [2] have this property.)

THEOREM 4.1. There is a unique member of $\mathscr{F}_{1}$ for each choice of the pair $\alpha, \lambda, 0<\alpha<\pi, 0<\lambda<\infty$. For fixed $\alpha$ the product $\kappa$ is a continuous strictly decreasing function of $\lambda$ while $\mu$ and $\nu$ are continuous strictly increasing functions of $\lambda$.

Proof. Existence of at least one member of $\mathscr{F}_{1}$ for given $\alpha, \lambda$. has been established in [2]. Suppose there are two such flows having values $\kappa_{1}, \nu_{1}, \kappa_{2}, \nu_{2}$ of $\kappa, \nu$, respectively. We may suppose $\nu_{1} \geqq \nu_{2}$. By Lemma $4.1, \kappa_{1} \leqq \kappa_{2}$. But equation (1.6) yields

$$
\lambda=\nu_{1} / \kappa_{1}^{3} \geqq \nu_{2} / \kappa_{2}^{3}=\lambda \text {. }
$$

Hence equality holds and the flows are identical by Lemma 4.1.

A similar argument shows that $\nu$ is a strictly increasing function of $\lambda$, while $\kappa$ is strictly decreasing. These functions are continuous. since any discontinuity in $\nu$ or $\kappa$ is reflected in a corresponding discontinuity in $\lambda=\nu / \kappa^{3}$. Hence $\mu=\nu / \kappa^{2}$ is also continuous and strictly increasing with $\lambda$.

Theorem 1.1 and its corollary Theorem 1.2 now follow directly from Theorem 4.1, as does the fact that $\kappa, \lambda, \mu, \nu$ are continuous: monotonic functions of one another.

Not only are these particular parameters continuous functions of each other; in a sense the flow as a whole depends continuously upon any one of them. To make this assertion precise, recall that in [2] 
the flows are expressed in terms of functions $u(t)$ defined on the first quadrant of the unit disk. In units for which $A=D=1$, the functions

$$
\begin{gathered}
w(t)=\kappa u^{\beta}(t) \\
z(t)=-1+\int_{1}^{t}\left[W^{\prime}(s) / w(s)\right] d s
\end{gathered}
$$

where

$$
\beta=2(\pi-\alpha) / \pi, W^{\prime}(s)=(2 / \pi s)\left(1-s^{2}\right) /\left(1+s^{2}\right)
$$

map this quarter-disk onto the hodograph and flow domains, respectively. The solutions $u(t)$ are obtained in [2], $\S 10,11$ as limits of locally uniformly bounded sequences of auxiliary functions. The same arguments may be used to show that the sequence $\left\{u_{n}\right\}$ corresponding to any bounded sequence $\left\{\lambda_{n}\right\}$ is locally uniformly bounded. Since the solution $u$ corresponding to each value of $\lambda$ is unique, it follows that if $\left\{\lambda_{n}\right\}$ converges to $\lambda$ then $\left\{u_{n}\right\}$ converges to $u$ uniformly on closed subsets of the quarter-disk excluding $t=i$. This yields

THEOREM 4.2. For fixed $\alpha$ the functions $w(t), z(t)$ of equations (4.2) are continuous with respect to the parameter $\lambda$ in the topology of uniform convergence on compact subsets of the quarter-circle with $t=0, i$ excluded.

With regard to monotonicity of the flows in the large we confine our remarks to monotonicity of the flow domain. This is best described in terms of the family $\mathscr{F}_{2}$ obtained by taking $A / q$ and $A / q^{2}$ as units of length and time, respectively, so that $A=q=1, D=\kappa, g=\lambda$, and equations (1.2), (1.3) become

$$
\begin{gathered}
v^{2}=1+2 \lambda h \\
x y^{2} \sim(2 \lambda \cos \alpha)^{-1} .
\end{gathered}
$$

THEOREM 4.3. For fixed $\alpha$ the domains of flows in $\mathscr{F}_{2}$ decrease, in the sense of set inclusion, with increasing $\lambda$.

Proof. Consider two flows in $\mathscr{F}_{2}$ having equal values of $\alpha$, free boundaries $\Gamma_{1}, \Gamma_{2}$, and parameter values $\kappa_{1}, \kappa_{2}, \lambda_{1}, \lambda_{2}$, respectively, where $\lambda_{1}>\lambda_{2}$. Let $r=r_{1}(\theta), r=r_{2}(\theta),-\alpha \leqq \theta<0$ be the polar equations of $\Gamma_{1}, \Gamma_{2}$. We are to show that the ratio $\rho(\theta)=r_{1}(\theta) / r_{2}(\theta)$ satisfies $\rho(\theta)<1$ for all values of $\theta$. This is true for the limits $\theta=-\alpha, 0$ since $\rho(-\alpha)=\kappa_{1} / \kappa_{2}<1$ by Lemma 4.1, and by equation (4.5) $\rho(\theta) \rightarrow$ $\left(\lambda_{2} / \lambda_{1}\right)^{1 / 3}<1$ as $\theta \rightarrow 0$. Finally one shows, by an argument paralleling the proof of Lemma 4.1, that $\rho_{0}=\sup \rho(\theta)$ satisfies $\rho_{0}<1$. 
5. Parameter ranges; limiting flows. The principal result of [2] is that the parameters $\alpha, \lambda$ for flows in $\mathscr{F}$ range independently over the respective intervals $(0, \pi)$ and $(0, \infty)$. For fixed $\alpha$ the ranges of $\kappa, \mu, \nu$ must also be intervals, possibly depending on $\alpha$, since by Theorem 4.1 these products are continuous monotonic functions of $\lambda$. We now prove

THEOREM 5.1. For fixed $\alpha$ the parameters $\lambda, \mu, \nu$ all have the: range $(0, \infty)$, while $\kappa$ is limited to the interval $\left(\kappa_{\infty}, \kappa_{0}\right), 0<\kappa_{\infty}<\kappa_{0}<\infty$, given by

$$
\begin{aligned}
\kappa_{\infty} & =(\pi-\alpha)^{-1} \\
\kappa_{0} & =(\sin \alpha)^{-1}\left[1+\frac{2}{\pi} \int_{0}^{\pi / 2} \tan \theta \sin (\alpha+\beta \theta) d \theta\right], \\
\beta & =2(\pi-\alpha) / \pi
\end{aligned}
$$

The values $\kappa_{\infty}, \kappa_{0}$ correspond to limiting flows as follows: $\kappa_{\infty}$ is the value of $\kappa$ for the "wedge flows" in the infinite sector lying above $H$ and to the left of $L$, having sources at infinity and sinks at 0 , while $\kappa_{0}$ corresponds to the class of Helmholtz flows (constant free boundary speed) satisfying conditions $(a)-(d)$ of $\S 1$.

Proof. Consider first the behavior for small values of $\lambda$. The Helmholtz flows satisfying (a)-(d) form a similarity class having circular sector hodographs [1]. In units for which $A=D=1$, they are represented by the dimensionless flow obtained by taking $u(t) \stackrel{\prime}{=} u_{0}(t) \equiv t$. in equations (4.2), i.e., by

$$
\begin{aligned}
& w_{0}(t)=\kappa_{0} t^{\beta} \\
& z_{0}(t)=-1+\kappa_{0}^{-1} \int_{1}^{t} W^{\prime}(s) s^{-\beta} d s .
\end{aligned}
$$

Here $\kappa_{0}$ is determined by the condition that $z_{0}$ maps the positive imaginary radius onto $L$. Since the normal distance from the slot-edge to $L$ is $\sin \alpha$, we have

$$
\kappa_{0}=-(\sin \alpha)^{-1} \operatorname{Im}\left\{e^{-i \alpha} \int_{\sigma} W^{\prime}(s) s^{-\beta} d s\right\}
$$

where $C$ is any path in the quarter-disk from $t=1$ to the imaginary radius, avoiding $t=0, i$. The expression (5.2) is obtained by deforming $C$ to run up the unit circumference.

Now the function $u_{0}(t) \equiv t$ is clearly the solution of the reduced problem in [2] for the limiting case $\lambda=0$. Moreover one may show as in the proof of Theorem 4.2 that as $\lambda$ approaches zero the solution. 
$u$ approaches $u_{0}$ uniformly on the quarter-disk, excluding $t=i$. Hence $\kappa$, which is given by

$$
\kappa=-(\sin \alpha)^{-1} \operatorname{Im}\left\{e^{-i \alpha} \int_{\sigma}\left[W^{\prime}(s) / u^{\beta}(s)\right] d s\right\}
$$

approaches $\kappa_{0}$ as $\lambda \rightarrow 0$, and (5.3) represents the limiting flow for small $\lambda$.

Turning now to the case of large $\lambda$, recall the proof of Lemma 3.1, where flows in $\mathscr{F}_{1}$ were compared with the wedge flow of unit flux. Since the point of maximum height of $\Gamma$ lies at the slot edge, we may set $v=\kappa, d=1$ in (3.1) to obtain

$$
\kappa>\kappa_{\infty},
$$

To show that $\kappa_{\infty}$ is in fact the lower limit on $\kappa$, and to study the limiting flow shape for large $\lambda$, consider again the family $\mathscr{F}_{2}$ of $\S 4$, consisting of dimensionless flows with $A=q=1$ and slot widths $\kappa$. We shall exhibit special flows which "approach" the wedge flow of unit flux, and use them to show that the members of $\mathscr{F}_{2}$ are equally close to this limiting flow when $\lambda$ is large.

In units for which $A=q=1$ the Helmholtz flow (5.3) becomes

$$
\begin{aligned}
& w_{1}(t)=t^{\beta} \\
& z_{1}(t)=-\kappa_{0}+\int_{1}^{t} W^{\prime}(s) s^{-\beta} d s .
\end{aligned}
$$

Let this flow be contracted uniformly about $O$ by the factor $a \gg 1$, so $w_{1}$ and $z_{1}$ are replaced by

$$
\begin{aligned}
& w_{a}(t)=a t^{\beta} \\
& z_{a}(t)=-\kappa_{0} a^{-1}+a^{-1} \int_{1}^{t} W^{\prime}(s) s^{-\beta} d s .
\end{aligned}
$$

Let $z=-\kappa_{a}$ be the point at which this contracted flow has velocity 1 , corresponding to $t_{a}=a^{-1 / \beta}$. Evaluating $\kappa_{a}=-z_{a}\left(t_{a}\right)$ asymptotically as $a \rightarrow \infty$, using (4.3), we find

$$
\kappa_{a}=\kappa_{\infty}+O\left(a^{-1}\right) \text {. }
$$

Finally we perturb this contracted Helmholtz flow by removing from its hodograph a half-disk of radius $\varepsilon \ll 1$ with center at $1+\varepsilon$. Let $w_{\varepsilon}(t)$ map the unit quarter-disk conformally onto this perturbed hodograph domain so that $w_{\varepsilon}$ agrees with $w_{a}$ at $t=0,1, i$. Then $w_{\varepsilon}$ approaches $w_{a}$ uniformly on the quarter-disk as $\varepsilon \rightarrow 0$ (as may be seen by applying Rado's theorem, [2] $\S 7$, after extending $w_{\varepsilon}^{1 / \beta}$ by reflection onto the whole disk.) The function 


$$
z_{\varepsilon}(t)=-\kappa_{\varepsilon}+\int_{t_{\varepsilon}}^{t}\left[W^{\prime}(s) / w_{\varepsilon}(s)\right] d s
$$

maps the quarter-disk unto this flow, where $t_{\varepsilon}$ is the real point at which $w_{\varepsilon}\left(t_{\varepsilon}\right)=1$ and $\kappa_{\varepsilon}$ is determined so that $z_{\varepsilon}$ maps the imaginary segment onto $L$. The hodograph and flow domains are illustrated in Figure 4. The dip in the horizontal boundary corresponds to the
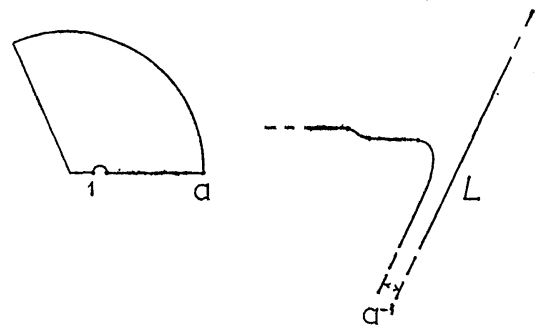

Fig. 4

indentation in the hodograph. Following this dip is a horizontal segment extending almost to $L$ (to $z=-\kappa_{0} / \alpha$ approximately). This joins the free boundary of constant flow speed $a$, which is asymptotic to the line parallel to and on the left of $L$, at a distance $a^{-1}$ from it. Clearly $\kappa_{\varepsilon} \rightarrow \kappa_{a}$ as $\varepsilon \rightarrow 0$, so that

$$
\kappa_{\varepsilon}=\kappa_{\infty}+o(1) \text { as } \varepsilon \rightarrow 0, a \rightarrow \infty \text {. }
$$

Furthermore the domain $\mathscr{F}_{\varepsilon}$ of this flow "approaches" that of the wedge flow, as a set-theoretic limit, as $\varepsilon \rightarrow 0, a \rightarrow \infty$.

Let this special flow be denoted by $\boldsymbol{F}_{\varepsilon}$, and let $\boldsymbol{F}$ denote a flow in $\mathscr{F}_{2}$ corresponding to a large value of $\lambda$. We assert that if $\lambda$ is sufficiently large, the domain $\mathscr{D}$ of $\boldsymbol{F}$ is contained in the domain $\mathscr{D}_{\varepsilon}$ of $\boldsymbol{F}_{\varepsilon}$. Hence the slot-width $\kappa$ of $\boldsymbol{F}$ satisfies $\kappa_{\varepsilon} \geqq \kappa>\kappa_{\infty}$, and $\kappa$ is as close to $\kappa_{\infty}$ as $\kappa_{\varepsilon}$.

Actually we will show, for every real number $r>1$, that if $\lambda$ is sufficiently large the domain obtained by contracting $\mathscr{D}$ about $O$ by the factor $r$ lies in $\mathscr{D}_{\varepsilon}$. The assertion then follows by applying this result to a second flow $\boldsymbol{F}_{\varepsilon^{\prime}}$ corresponding to a larger value of $a$ and a smaller value of $\varepsilon$, taking $r$ to be the greatest number for which the expansion of $\mathscr{D}_{\varepsilon^{\prime}}$ about $O$ by the factor $r$ remains inside $\mathscr{D}_{\varepsilon}$.

Suppose $\mathscr{D}$ is not already inside $\mathscr{D}_{\varepsilon}$. Then there is a number $\rho>1$ such that when $\boldsymbol{F}$ is contracted by the factor $\rho$ the contracted domain is inside $\mathscr{D}_{\varepsilon}$, the two curved boundaries being tangent at some point $N$. Let $v, h$ be the speed of $\boldsymbol{F}$ and depth, respectively, at the point on $\Gamma$ which goes into $N$ under the contraction, and $v_{\varepsilon}, h_{\varepsilon}$ be the speed of $\boldsymbol{F}_{\varepsilon}$ and depth at $N$. By Theorem 2.1 (b), $v_{\varepsilon}>\rho v$. Combining 
with (4.4) and $v_{\varepsilon} \leqq a, h=\rho h_{\varepsilon}, \rho>1$, we obtain $h_{\varepsilon}<a^{2} / \lambda$. This implies that $N$ is near the slot edge of $\boldsymbol{F}_{\varepsilon}$ when $\lambda$ is large, so that $v_{\varepsilon}$ is near the value 1 . Since $v>1$ we also have $\rho<v_{\varepsilon} \rightarrow 1$. Hence $\rho<r$ when $\lambda$ is sufficiently large, and the assertion follows.

The proof of Theorem 5.1 is completed with the observation that $\mu$ and $\nu$ share with $\lambda$ the range $(0, \infty)$, in view of the above bounds on $\kappa$ and the identities $\mu=\kappa \lambda, \nu=\kappa^{3} \lambda$.

\section{REFERENCES}

1. G. Birkhoff and E. H. Zarantonello, Jets, wakes, and cavities, Academic Press, New York (1957), Ch. II.

2. D. S. Carter, Existence of a class of steady plane gravity flows, Pacific J. Math., 11 (1961), 803-819.

3. - Local behavior of plane gravity flows at the confluence of free boundaries and analytic fixed boundaries, J. Math. Mech., 10 (1961) 441-450.

4. - Local behavior of plane gravity flows. II. Infinite jets on fixed boundaries, J. Math. Mech., 13 (1964), 329-352.

5. H. Lewy, $A$ note on harmonic functions and a hydrodynamical application, Proc. Amer. Math. Soc., 3 (1952), 111-113.

6. - Developments at the confluence of analytic boundary conditions, Univ. of Calif. Publications in Math., New Series, 1 (1950), 247-280.

7. James B. Serrin, Jr., Uniqueness theorems for two free boundary problems, Amer. J. Math., 74 (1952), 492-506.

8. - Two hydrodynamic comparison theorems, J. Math. Mech., 1 (1952), 563-572.

9. - On plane and axially symmetric free boundary problems, J. Math. Mech., 2 (1953), 563-575.

OREGON STATE UNIVERSITY 
